\title{
How Should Transcranial Direct Current Stimulation be Used in Populations With Severe Alcohol Use Disorder? A Clinically Oriented Systematic Review
}

Clinical EEG and Neuroscience

$\mathrm{I}-17$

(C) EEG and Clinical Neuroscience

Society (ECNS) 2021

Article reuse guidelines:

sagepub.com/journals-permissions

DOI: $10.1177 / 15500594211001212$

journals.sagepub.com/home/eeg

(3)SAGE

\author{
Zoé Bollen' (iD, Valérie Dormal', and Pierre Maurage' (D)
}

\begin{abstract}
Background and rationale. Severe alcohol use disorder (SAUD) is a major public health concern, given its massive individual, interpersonal, and societal consequences. The available prevention and treatment programs have proven limited effectiveness, as relapse rates are still high in this clinical population. Developing effective interventions reducing the appearance and persistence of SAUD thus constitutes an experimental and clinical priority. Among the new therapeutic approaches, there is a growing interest for noninvasive neuromodulation techniques, and particularly for transcranial direct current stimulation (tDCS) as an adjunctive treatment in neuropsychiatric disorders, including SAUD. Methods. We propose a systematic review, based on preferred reporting items for systematic reviews and meta-analyses (PRISMA) guidelines, evaluating the available evidence on the effectiveness of tDCS to improve clinical interventions in SAUD. Results. We provide an integrative overview of studies applying tDCS in clinical populations with SAUD, together with a standardized methodological quality assessment. We show that the currently available data remain inconsistent. Some data suggested that tDCS can (I) reduce craving, relapse or alcohol-cue reactivity and (2) improve cognitive control and inhibition. However, other studies did not observe any beneficial effect of tDCS in SAUD. Conclusions. Capitalizing on the identified strengths and shortcomings of available results, we present evidence-based clinical guidelines to integrate tDCS in current clinical settings and to combine it with neurocognitive training.
\end{abstract}

\section{Keywords}

alcohol, tDCS, neuromodulation, alcohol-use disorders, cognitive remediation

Received October 2, 2020; revised December 24, 2020; accepted February 12, 2021.

\section{Introduction}

Excessive alcohol use constitutes a major public health concern, ${ }^{1,2}$ leading to cognitive ${ }^{3}$ and cerebral $l^{4}$ impairments. These deleterious consequences are particularly widespread in severe alcohol use disorder (SAUD), ${ }^{5}$ which is among the most prevalent psychiatric conditions. ${ }^{6}$ The societal burden of SAUD is massive, particularly because of the limited access patients have to clinical structures, leading to a high treatment gap. ${ }^{7}$ Even when patients access clinical settings, high relapse rates are observed. ${ }^{8}$ There is thus an urgent need to improve the rehabilitation programs offered to patients, to bring new populations to clinical settings, and to treat these patients more efficiently. To address these issues, new therapeutic avenues have emerged, notably focusing on neurocognitive remediation. ${ }^{9,10}$ As cognitive impairments interfere with intervention outcomes and prognosis, ${ }^{11-17}$ preliminary studies have confirmed the benefits of neurocognitive training on treatment outcomes, relapse rate, and quality of life in SAUD. ${ }^{18-20}$

Neuromodulation techniques have recently arisen as complementary intervention tools, notably to enhance the outcome of cognitive remediation. These techniques improve cognitive functioning by targeting dysfunctional neuronal circuitry involved in addictive behaviors. ${ }^{21}$ Among these neuromodulation tools, transcranial direct current stimulation (tDCS) is considered as a promising avenue, since it allows the safe modulation of brain activity. ${ }^{22}$ Beyond its affordable cost, tDCS has no substantial side effects, contrary to other neuromodulation modalities. tDCS is based on a portable device composed of a battery and a pair of rubber electrodes, inducing a weak and direct electric current (usually $0.5-2 \mathrm{~mA}$ ), modifying the cortical excitability of the cerebral regions located under the electrodes. ${ }^{23}$ It induces plasticity by modulating neuronal resting membrane potentials according to electrode polarity: anodal stimulation increases cortical excitability while cathodal stimulation decreases it. ${ }^{24-26}$

\footnotetext{
'Louvain Experimental Psychopathology Research Group (LEP), Psychological Science Research Institute, UCLouvain, Louvain-la-Neuve, Belgium

Corresponding Author:

Pierre Maurage, UCLouvain, Faculté de Psychologie, Place du Cardinal Mercier 10, B-I348 Louvain-la-Neuve, Belgium.

Email: pierre.maurage@uclouvain.be
} 
The neuromodulation induced by tDCS allows manipulating a large range of cognitive processes in healthy populations, including motor/visual skills, language learning, or higher cognitive functions. ${ }^{27,28}$ By fostering neuroplasticity, tDCS also enhances rehabilitation in neuropsychiatric populations. ${ }^{29}$ The impact of tDCS has been widely investigated for the treatment of depression, ${ }^{30-32}$ schizophrenia, ${ }^{33,34}$ and Parkinson's disease, ${ }^{35,36}$ where it is now considered as a useful complementary tool to reduce psychopathological symptoms or increase cognitive abilities. However, its application is still restricted in substance abuse and only a few studies have been conducted to determine its clinical efficiency in SAUD.

Several papers have recently reviewed the available literature regarding the effect of tDCS on SAUD. Most reviews also encompassed other substance use disorders ${ }^{21,37,38}$ and/or other neuromodulation techniques, ${ }^{39-41}$ and were thus not focusing on tDCS in SAUD. Their main outcome regarding tDCS in SAUD is that the current literature has massive limitations, including low sample sizes, heterogeneity regarding stimulation parameters, and lack of long-term outcomes evaluation. ${ }^{42-44}$ Most reviews thus concluded that further experimental investigations are needed before applying tDCS in clinical contexts. This conclusion might result from three main shortcomings related to these review papers. First, none of them offered a sound and standardized methodological assessment of the published studies, hampering the determination of the empirical value and soundness of their conclusions. Second, they most often merged results coming from clinical and subclinical populations, thus preventing to identify the specific usefulness of tDCS in SAUD. Third, they were focused on research purposes, and their clinical perspectives were therefore limited. As a whole, beyond the ongoing theoretical debates on tDCS use in SAUD, no concrete guidelines exist regarding why, when, and how the implementation of tDCS in SAUD treatment can be done in clinical practice. This gap between experimental results and their clinical application is unfortunate for caregivers, who have the means and desire to include tDCS in their therapeutic programs, but cannot do so due to the absence of clinical guidelines. It is also deleterious for patients, who do not benefit from useful tDCS interventions.

We thus offer the first review focusing on the use of tDCS in SAUD. This systematic review, following preferred reporting items for systematic reviews and meta-analyses (PRISMA) guidelines, explores the effectiveness of tDCS as an intervention tool in SAUD, by comparing studies investigating tDCS among patients with SAUD and by assessing their methodological quality through a quality assessment tool. ${ }^{45}$ We also provide comprehensive guidelines for the clinical application of tDCS and investigate the advantages of using this technique in SAUD.

\section{Methods}

\section{Articles Identification and Selection Procedure}

This systematic review followed PRISMA guidelines. ${ }^{46}$ Three databases were consulted (PsycINFO, Pubmed, and Scopus).
The procedure, conducted independently by 2 authors (ZB and PM), focused on peer-reviewed articles published in English, between January 1, 2000 and September 4, 2020. The search phrase combined neurostimulation words ("tDCS" OR "transcranial direct current stimulation" OR "transcranial DC stimulation" OR "neuromodulation" OR "brain stimulation") and alcohol-related terms ("alcoholism" OR "alcohol dependence" OR "alcohol-use disorder" OR "severe alcoholuse disorder" OR "SAUD" OR "problematic drinking" OR "alcohol" OR "alcohol consumption"). The initial search identified 561 articles (222 in PsycINFO, 173 in Pubmed, and 166 in Scopus). The articles to be included were then selected according to a 3-step procedure (Figure 1). First, duplicates were removed, identifying 339 unique papers. Second, titles and abstracts were screened, and articles presenting one of the following exclusion criteria were removed: (1) no tDCS exposure, (2) no peer-reviewed experimental data presented, (3) no human sample, and (4) no valid substance use measure. When this screening did not allow a clear-cut decision, the paper was included in the full-text reading phase. This step led to the exclusion of 306 articles. Third, the 33 remaining articles were screened through full-text reading, leading to the exclusion of 21 articles, either presenting a case report, primarily focusing on other substance use or psychiatric/neurological disorders, or not focusing on a population with SAUD. Twelve articles were included in the review. The independent literature searches performed by both authors (ZB and PM) ended up in the selection of the same 12 articles, thus presenting a total coherence.

\section{Methodological Quality Assessment}

Studies were evaluated using the "quality assessment of controlled intervention studies" scale. ${ }^{45}$ The scale comprises 14 items with a binary answer (yes/no). For each study, a score (ie, percentage of items with a "yes" answer) was computed, leading to a global quality rating (ie, poor: $<50 \%$; fair: 50\%-69\%; good: $70 \%-79 \%$; strong: $80 \%$ and beyond). ${ }^{47}$ When the information contained in the paper did not allow to make a clear decision regarding an item, the mention "not reported" (NR) was used, and this item was excluded from score computation. To increase the procedure reliability, 2 independent judges (ZB and PM) performed the quality assessment. The total agreement between them was $95.2 \%$ (160 of 168 evaluation criteria), which can be considered as very high. Assessment discrepancies (related to items 4, 5, 7, 8 and 10) were then discussed with the second author (VD) to obtain a consensus. The Supplemental Table 1 reports this quality assessment.

\section{Data Extraction and Synthesis}

Six categories of variables were reported through a systematic data extraction procedure, adapted from the population, intervention, comparison, outcomes, study design (PICOS) protocol (Table 1): (1) participants, (2) exposures, (3) comparison, 


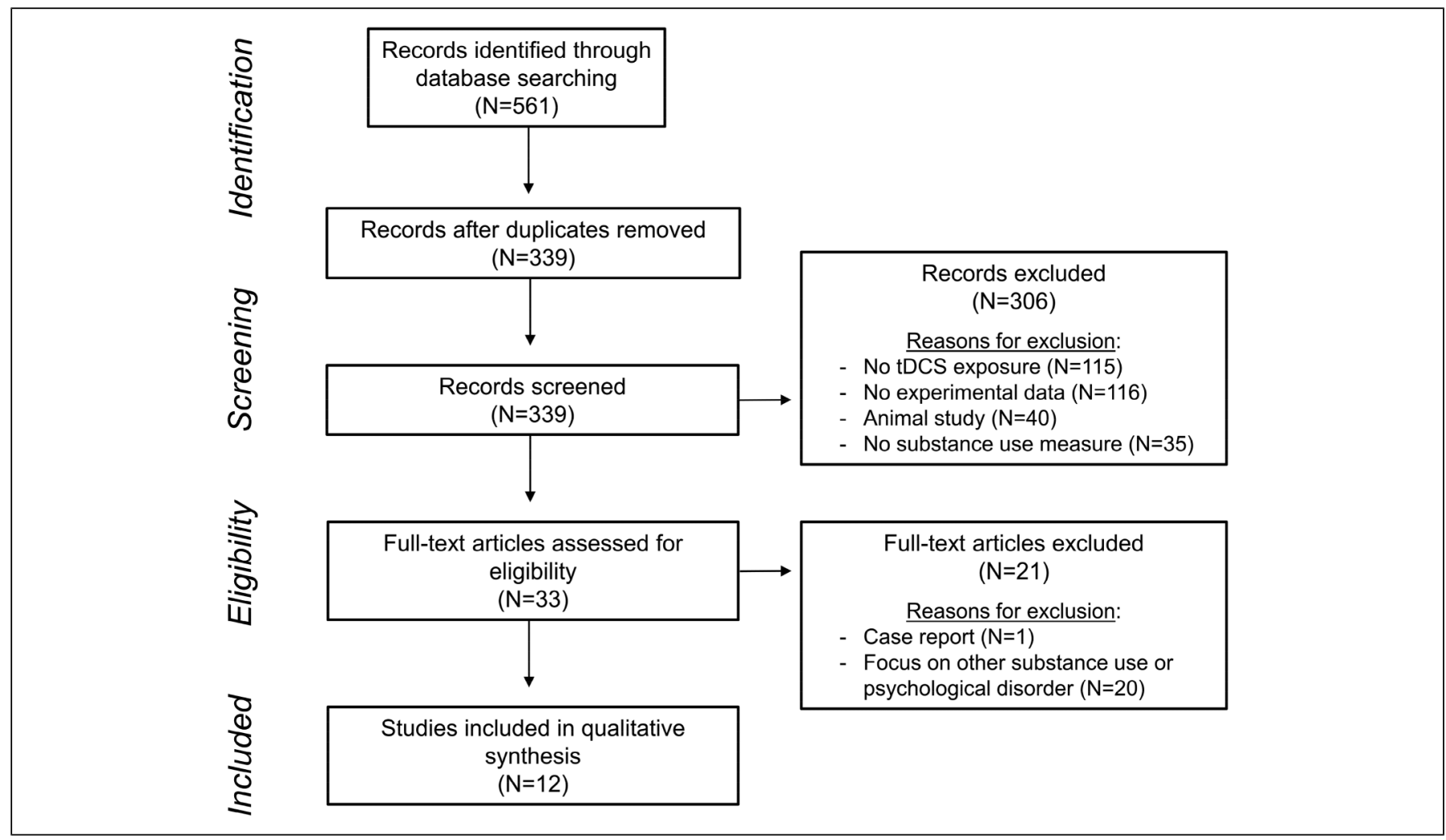

Figure I. PRISMA flow diagram describing the selection and review process of the papers included.

(4) intervention, (5) stimulation protocol, and (6) outcomes. The main outcomes of the included studies were described in two subsections, according to whether tDCS was applied as a stand-alone treatment or combined with cognitive interventions. This distinction was based on the hypothesis that the latter application would be more effective due to the synergistic benefits derived from this combination, as suggested by previous reviews. ${ }^{48}$

\section{Results}

\section{Quality Assessment}

Nine studies presented strong quality, 2 good quality and 1 fair quality. All studies showed good adherence to the tDCS protocols in all groups and did not report any major side effects. The implementation and assessment of clinical outcomes were good, all studies using reliable experimental tasks or questionnaires, and all participants were analyzed according to the group they were assigned to. Most studies considered biasing variables (eg, sociodemographic and alcohol use measures), and no significant group differences were shown regarding these variables at baseline. Moreover, most of them considered psychopathological comorbidities as exclusion criteria and 2 studies used a within-subject design that allows minimizing the impact of interindividual heterogeneity. The overall drop-out rate was low across studies and did not highly differ between treatment groups. Before conducting the clinical trial, most studies have generated hypotheses, reported the outcomes to be measured, and prespecified the different groups. Nine studies reported an adequate randomization method. Nevertheless, the main issues regarding tDCS studies concerned the double-blind assignments and the sample size. Indeed, in only 6 studies were the participants, providers, and assessors all blinded, and most studies did not capitalize on a priori power computations to determine the sample size or did not provide an effect size computation to estimate the strength of the statistical results obtained.

\section{Main Outcomes}

tDCS Applied as a Stand-alone Treatment. The first study using tDCS in SAUD explored its impact on craving. ${ }^{49}$ Craving was evaluated through the alcohol urge questionnaire $(\mathrm{AUQ})^{50}$ before and after being confronted with alcohol-related visual cues. They performed a single tDCS session on 13 recently detoxified patients. They used an offline approach (ie, stimulation at rest) in which tDCS effects were measured by comparing preassessment and postassessment craving scores. They reported that bilateral tDCS stimulation delivered to the dorsolateral prefrontal cortex (DLPFC), whatever the stimulation laterality, reduced cue-provoked craving. This finding was supported by neuroimaging studies that demonstrated the 


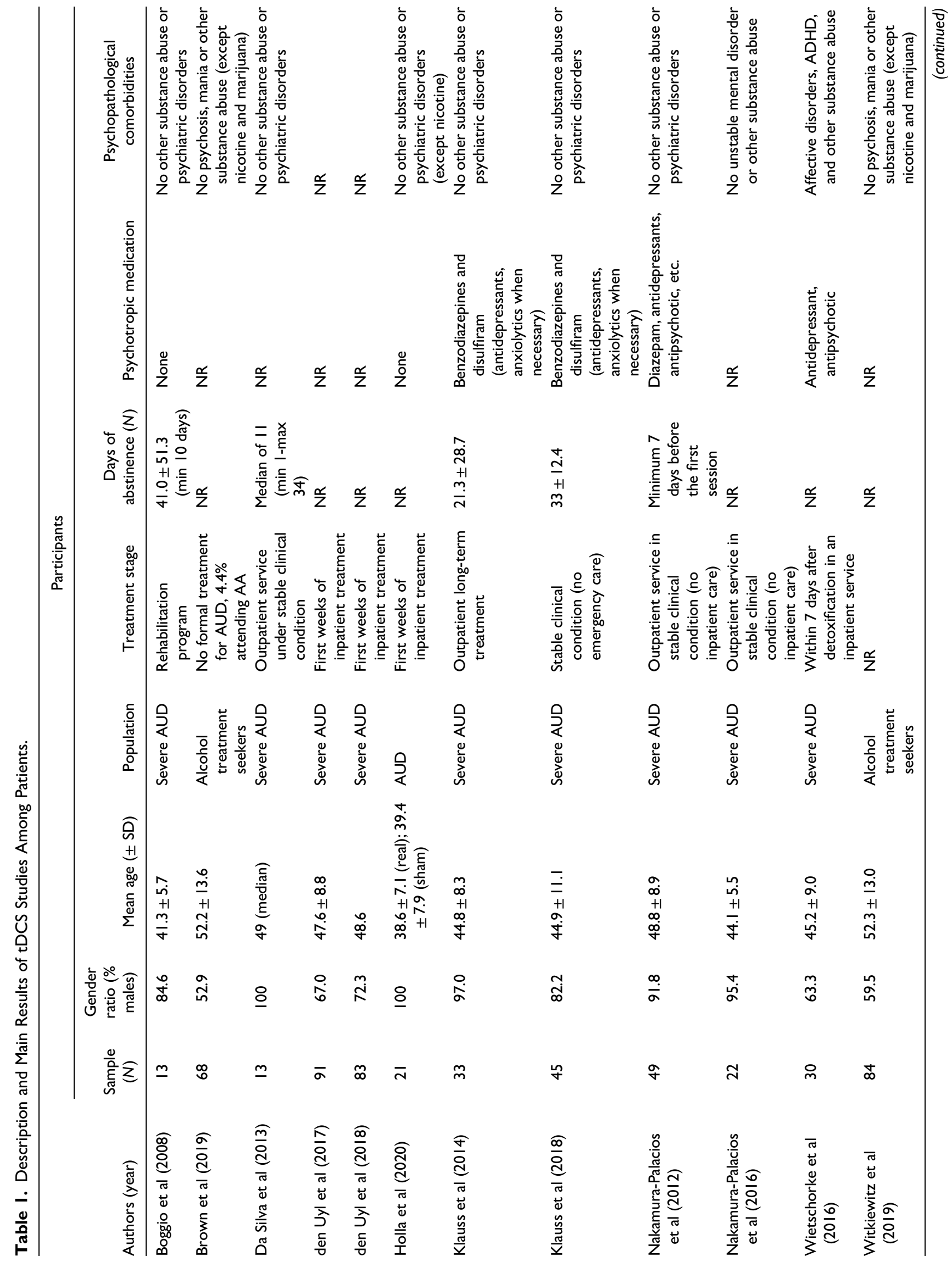




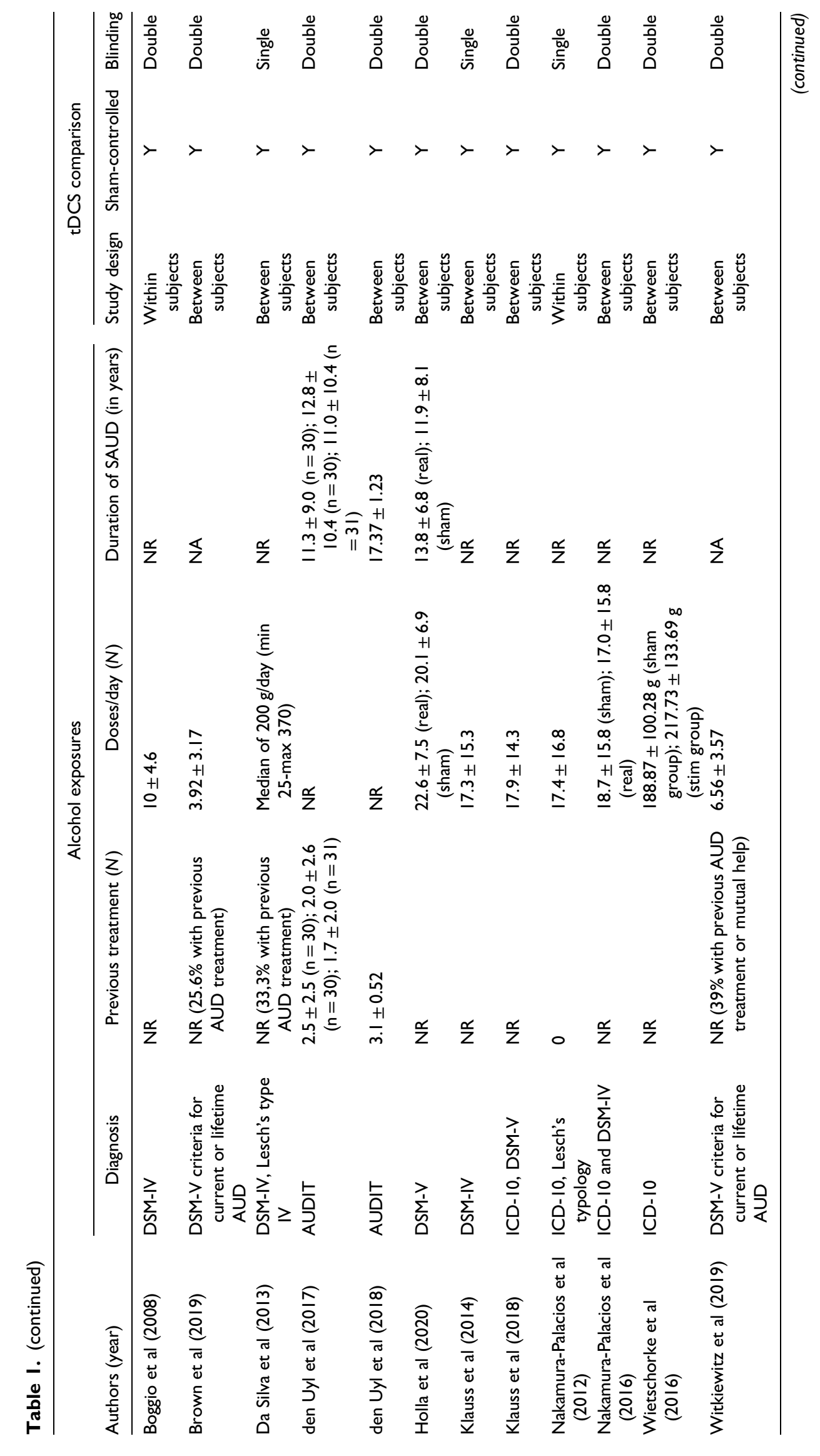




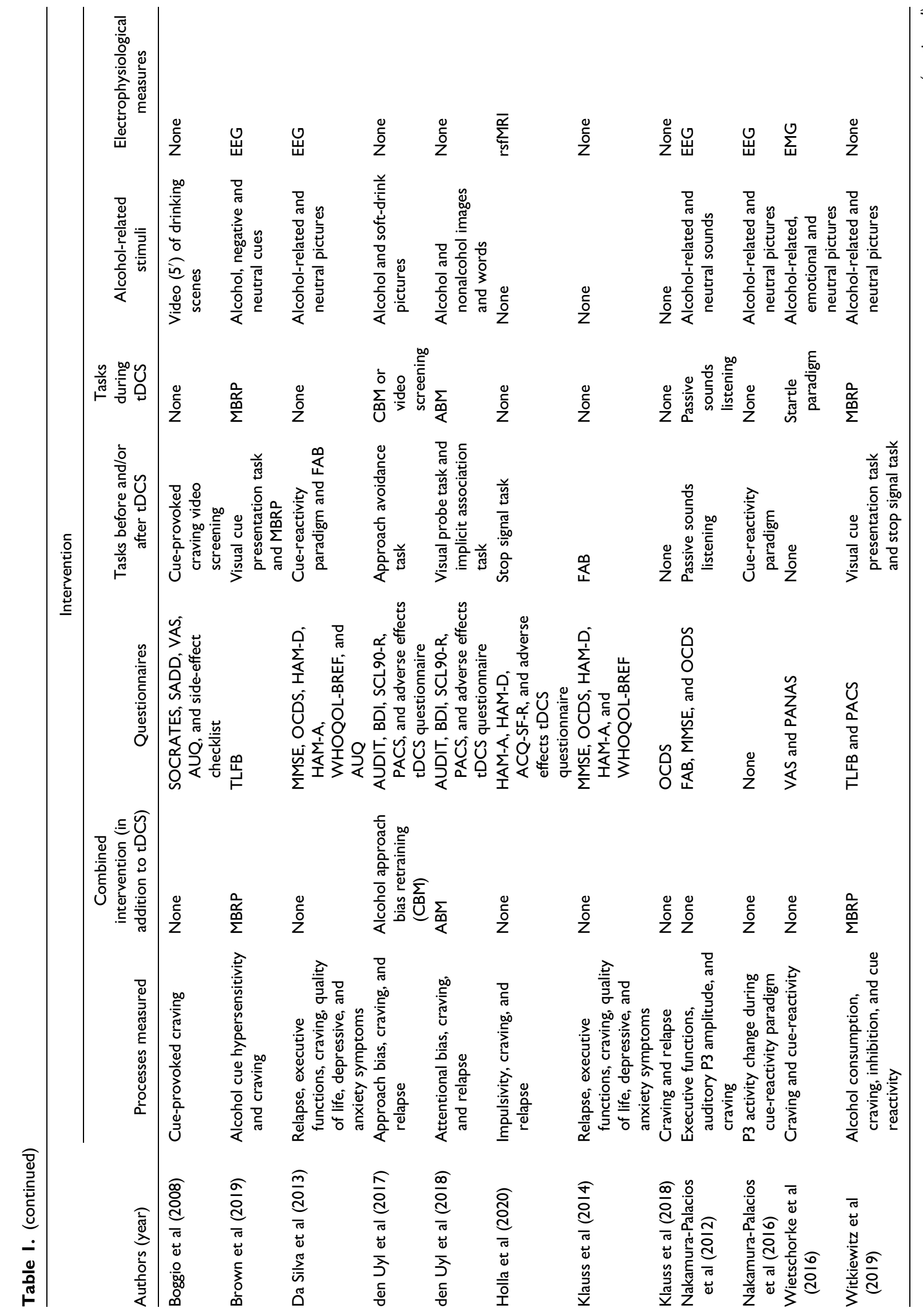




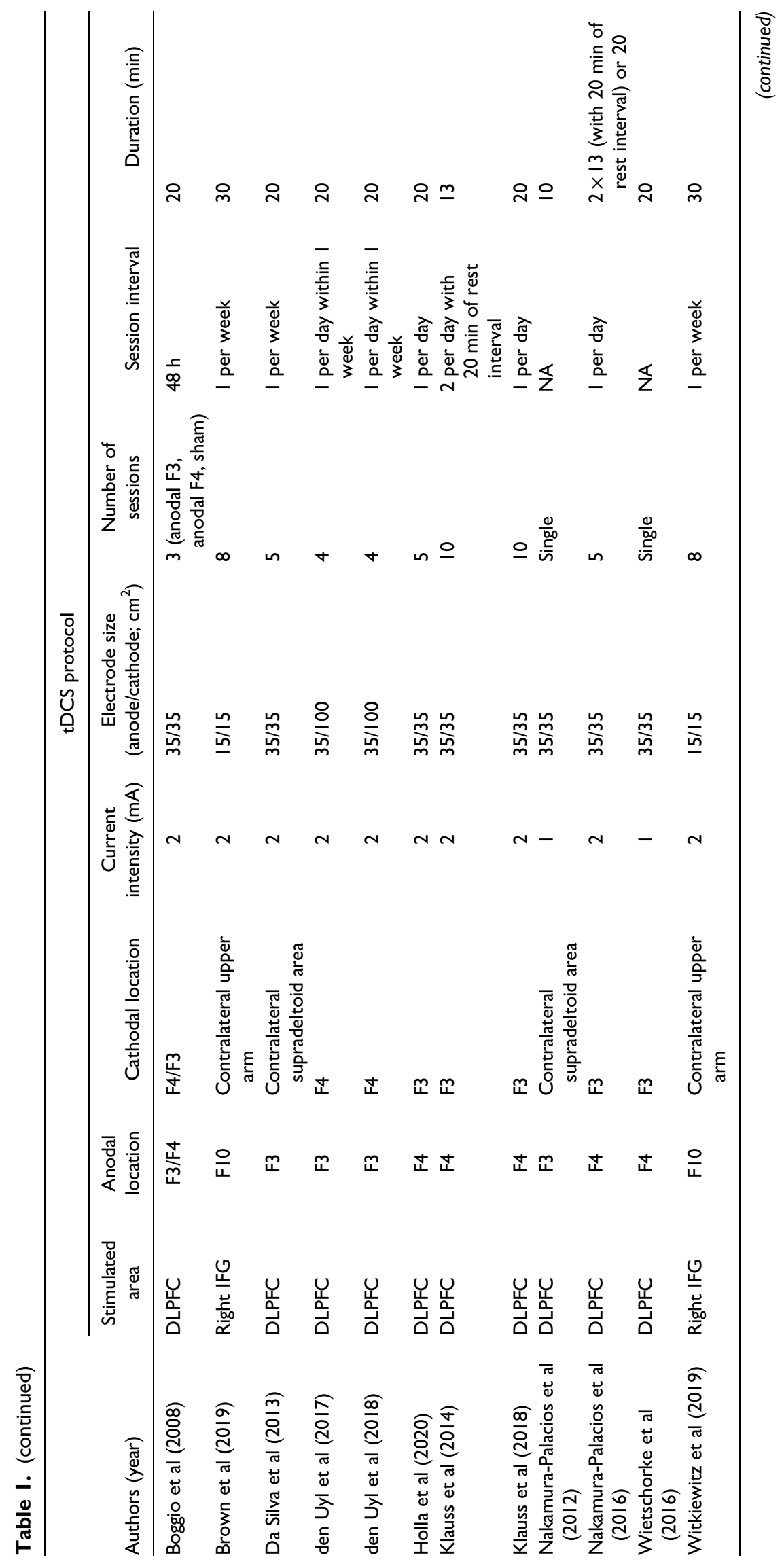




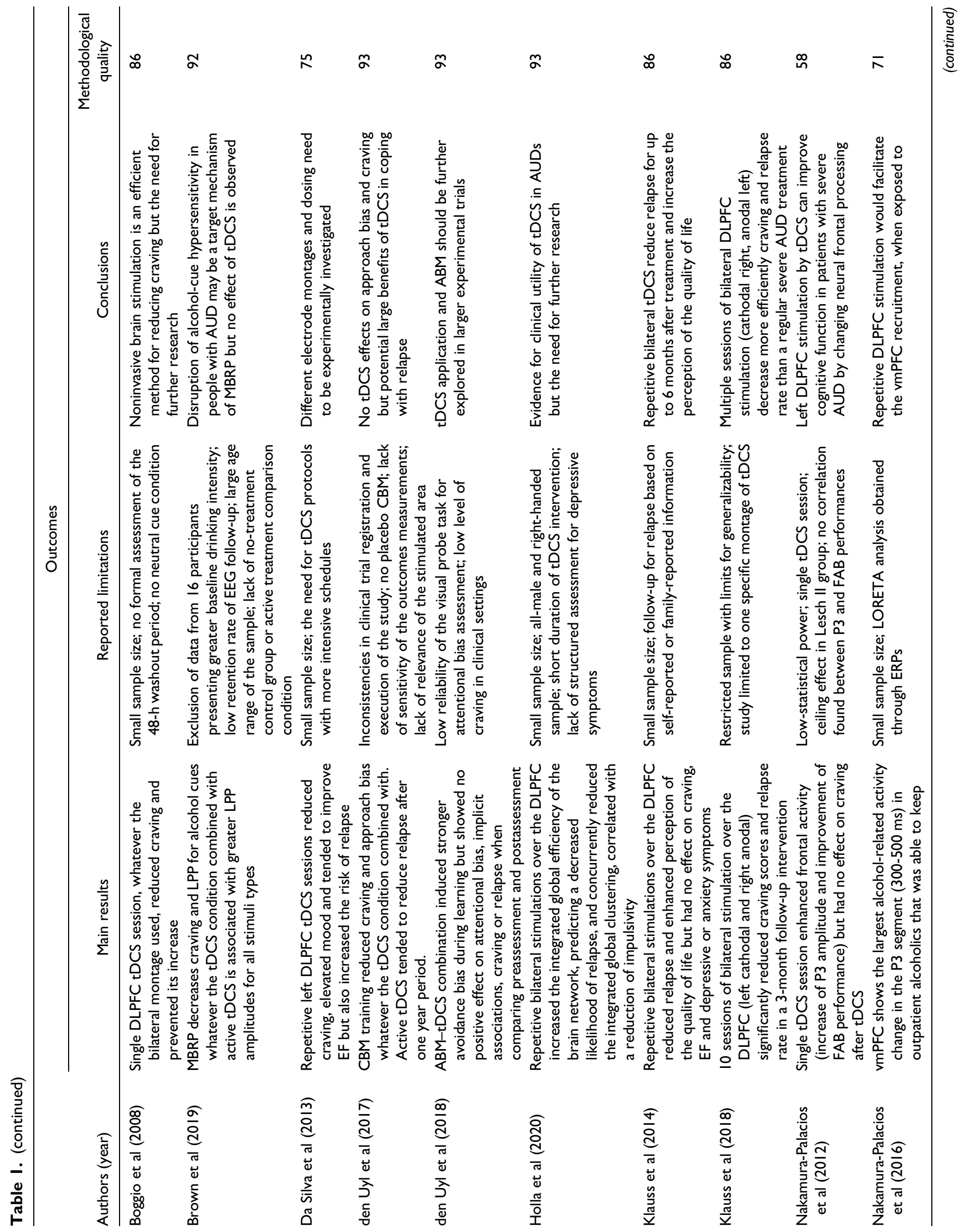




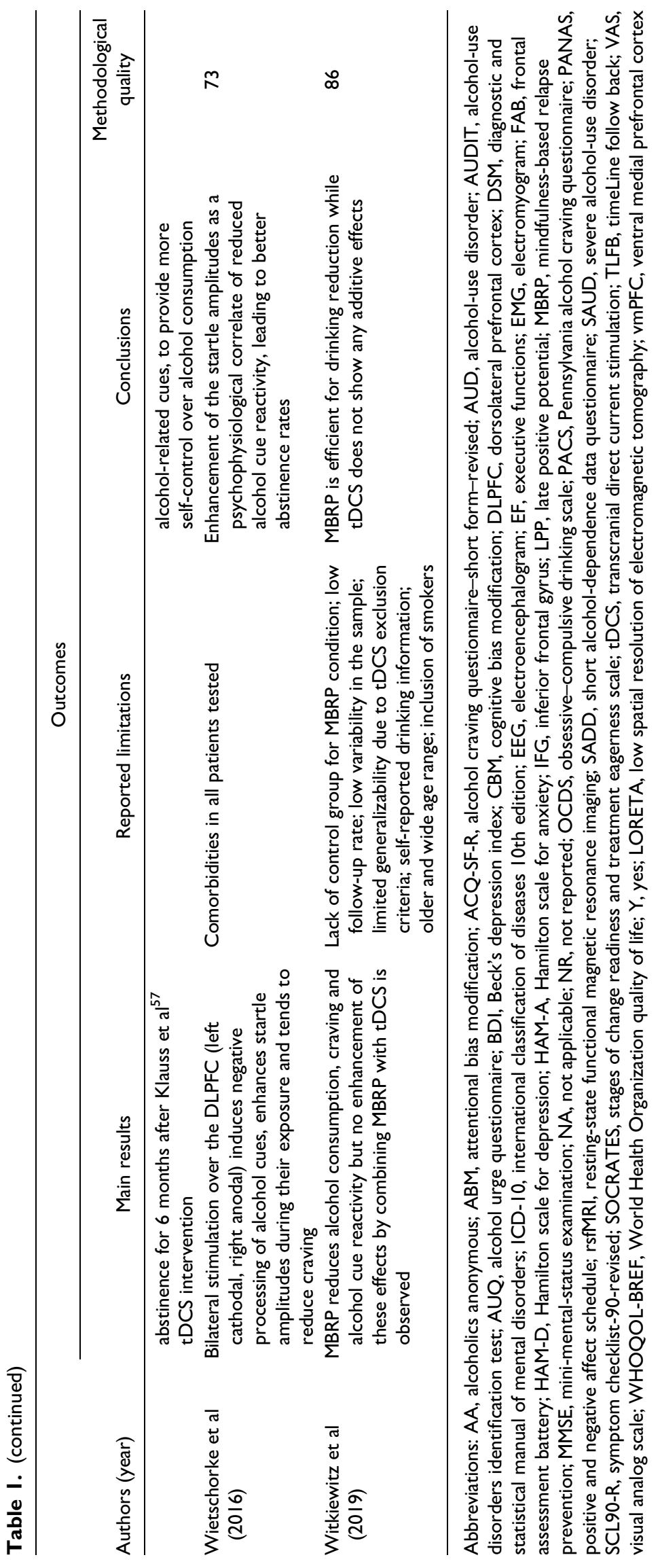


role of DLPFC in craving. ${ }^{51,52}$ These results suggested that both bilateral montages would preserve patients from craving states by disrupting the balance between right/left DLPFC activity. ${ }^{49}$ This study obtained a strong methodological quality score (86\%) and applied a within-subject design but the sample size was very small.

Nakamura-Palacios et $\mathrm{al}^{53}$ investigated the clinical and electrophysiological effects of tDCS on frontal lobe deficiency on 49 detoxified patients. During the presentation of alcohol-related and neutral sounds, the auditory P3 event-related potential (ERPs) was measured before, during, and after a single anodal tDCS over the left DLPFC. They evaluated frontal functions by the frontal assessment battery $(\mathrm{FAB})^{54}$ and subjective craving by a self-reported questionnaire. The subtype IV patients according to Lesch's typology showed behavioral (higher FAB performance) and electrophysiological (more pronounced P3 amplitude increase when confronted with alcoholrelated sound) frontal activity improvement after tDCS. This result was expected as this subtype regroups the most severely impaired patients (ie, presenting major executive dysfunction) and were thus the most likely to benefit from this treatment. Nevertheless, craving was not influenced by tDCS. While they obtained the lowest but fair methodological quality score (58\%), their sample size was quite large and they used a within-subject design with comprehensive measures of frontal activity.

A similar study replicated their electrode montage $e^{53}$ and investigated the behavioral and electrophysiological changes induced by multiple tDCS sessions. ${ }^{55}$ They examined the effects of left DLPFC modulation on relapse rate in 13 Lesch IV outpatients. Through 5 sessions, FAB performance ${ }^{54}$ and neural activation in frontal regions when confronted with alcohol-related and neutral visual cues were obtained in an offline approach, before and after tDCS stimulation. Craving, depressive, and anxiety symptoms, as well as the quality of life were measured through self-reported questionnaires. Repetitive tDCS stimulation improved executive functioning and was significantly linked to mood improvement and craving reduction at treatment end. However, anodal modulation of left DLPFC was related to increased relapse risk. Such paradoxical results pointed out that even if craving could generally facilitate relapse, it is not the only factor involved. ${ }^{55,56}$ Their sample size was small but their design was strengthened by multiple tDCS sessions, electrophysiological measures, and various outcome assessments, and they obtained a good quality score $(73 \%)$.

Since the anodal tDCS over the left DLPFC tended to increase relapses, ${ }^{55}$ Klauss et $\mathrm{al}^{57}$ hypothesized that the opposite effect would be observed by using inverse stimulation polarity (ie, cathodal tDCS over the left DLPFC). They replicated their methodological design ${ }^{55}$ and also investigated the potential use of a tDCS protocol with more intensive schedules (2 daily sessions for 5 consecutive days) in 33 outpatients. Bilateral tDCS over the DLPFC induced a significant relapse rate reduction: $50 \%$ of the stimulated group remained abstinent after the 6-month follow-up period compared with $11 \%$ in the placebo group. Although quality of life was enhanced, tDCS had no effect on depressive/anxiety symptoms, subjective craving or executive functioning. They obtained a strong quality score (93\%), applied multiple and intense tDCS sessions and assessed a large range of outcomes.

Among the patients who remained abstinent following stimulation in the study by Klauss et $a,^{57} 8$ took part in a study investigating the electrophysiological effects of repetitive bilateral tDCS over the DLPFC in alcohol and crack-cocaine addiction. ${ }^{58}$ The study explored, through electroencephalogram (EEG), the brain region with the highest change after tDCS treatment in the P3 temporal segment $(300-500 \mathrm{~ms})$ in a cue-reactivity paradigm. Eight abstinent patients received real-tDCS with the same electrode montage used by Nakamura-Palacios et al, ${ }^{53}$ while 14 patients who previously relapsed were enrolled in the sham-controlled group. The analysis of the $\mathrm{P} 3$ segment identified the ventral medial prefrontal cortex (vmPFC) as the region with the largest activation changes during the presentation of alcohol-related cues. As its activity is related to self-control behaviors and bottom-up-driven evaluating process, ${ }^{59-61}$ DLPFC stimulation could facilitate the vmPFC recruitment to provide more controlled behaviors when exposed to alcohol-related cues. Their sample size was small but their methodological quality was good (79\%) and they used electrophysiological measures, offering complementary insights on tDCS effects.

The same research group further investigated whether extended use of the same tDCS intervention during 10 consecutive days could result in a more effective reduction of craving and relapse. ${ }^{62}$ The same stimulation parameters were used, although tDCS sessions were applied once a day during a 20 -min period. They tested 45 patients in a randomized, double-blind clinical trial in which they conducted subjective craving measurements before, during, and after tDCS. Their results supported the efficiency of intensive tDCS sessions as adjunctive therapy, since craving scores progressively decreased during the real-tDCS treatment compared with the sham-tDCS group. Moreover, the 3-month follow-up revealed an abstinence rate of $72.7 \%$ in the real-tDCS group compared with $27.8 \%$ in the sham-tDCS group. The strengths of this study are the high frequency of tDCS sessions and its strong quality score (93\%), while its weakness concerns the absence of behavioral or electrophysiological measures.

Wietschorke et $\mathrm{al}^{63}$ explored the underlying mechanisms of bilateral tDCS (left cathodal/right anodal) by examining the change in cue-reactivity and subjective craving induced by this stimulation in 30 detoxified patients. In a single session, subjective craving was measured using visual analog scales and the reactivity to alcohol-related/emotional pictures was measured through the startle reflex paradigm, by using an electromyogram (EMG) placed on the eyelid closure. ${ }^{64}$ The immediate tDCS effects were evaluated by using an online approach. As predicted, bilateral tDCS over the DLPFC induced more negative processing of alcohol cues, increasing startle amplitudes during alcohol-related stimuli exposure, and finally resulting in a trend toward craving reduction. Startle amplitudes 
would thus be an efficient quantifiable measure of alcohol cue reactivity. Wietschorke et $\mathrm{al}^{63}$ argued that this psychophysiological correlate might be one of the main mechanisms leading to a reduced relapse rate. Their strengths are the use of EMG based on an online approach and their methodological quality score of $83 \%$.

Finally, Holla et $\mathrm{al}^{65}$ explored the effects of multiple offline tDCS sessions on resting-state functional magnetic resonance imaging (rsfMRI), as well as its correlates with impulsivity and relapse. Twenty-one patients were allocated to either 5 real or sham bilateral (left cathodal/right anodal) tDCS sessions over the DLPFC. At 2 time-points (baseline and postintervention), they completed the 10-min rsfMRI scan and performed a stopsignal task to assess impulsivity. The authors showed an increase in global efficiency (ie, brain networks integration) following tDCS sessions, as well as a reduction in global clustering (ie, brain networks segregation). The former finding allowed us to predict a reduced relapse risk while the latter was correlated with decreased impulsivity after tDCS. They also reported an increase in the functional connectivity of sub-network related to prefrontal regions. This study shows a strong quality score $(93 \%)$ and multiple sessions design with the use of rsfMRI.

tDCS Combined with Cognitive Interventions. Some researchers examined whether anodal stimulation over the left DLPFC improves the retraining of the alcohol approach and attentional biases, ${ }^{66,67}$ such retraining having beneficial effects in SAUD. ${ }^{68,69}$ In den Uyl et al's study, ${ }^{66} 91$ patients performed 4 approach-avoidance task sessions. During the training version, pictures of alcohol beverages were systematically presented on the avoidance side (pictures of soft drinks appearing on the approach side). For the assessment version, the alcohol and softdrink pictures were presented with an equal probability on both sides, while participants received either real or sham-tDCS. Results showed a decrease of craving and approach bias scores over time (either due to cognitive bias modification [CBM] training and/or learning effects), but the combination of tDCS with cognitive training did not enhance these effects. While no effect was found on relapse after the 3-month follow-up, a trend toward reduced relapse rates was observed after 1 year in patients who received tDCS combined with alcohol approach bias retraining. Den Uyl et $\mathrm{al}^{67}$ also investigated whether tDCS could enhance attentional bias modification (ABM) training by assigning 98 patients into 4 groups that followed a $2 \times 2$ factorial design. Groups received 4 sessions of control/ real ABM training, combined with real/sham-tDCS. In a modified visual probe task, participants responded to a probe appearing at the same location as 1 of the 2 previous stimuli (ie, alcohol or nonalcohol-related picture). In the training version, $90 \%$ of the probes appeared after the nonalcohol cues, while the control and assessment version kept an equivalent contingency. They tested the possible improvement of ABM effects by tDCS on implicit alcohol bias, craving, and relapse rates. Although the results from their online measures (ie, during training) showed enhanced learning with stronger avoidance bias in the real ABM training with the real-tDCS group, no positive effects of the ABMtDCS combination were observed on the attentional bias, craving or relapse. The strengths of both studies are their large sample size and their excellent quality score (93\%) while their weakness concerns the use of retraining programs recruiting a low level of cognitive resources.

The benefits derived from pairing tDCS with cognitive interventions were further explored by 2 studies combining tDCS over the right IFG with mindfulness-based relapse prevention (MBRP), defined as a clinical intervention combining the cognitive-behavioral skills with mindfulness practices. ${ }^{70,71}$ Witkiewitz et al $^{70}$ conducted their study on 84 individuals with current or lifetime AUD, interested in reducing drinking. They assessed alcohol consumption, craving, alcohol cue reactivity, and response inhibition through self-reported questionnaires and tasks (ie, visual cue presentation task and stop-signal task) at baseline, directly after treatment and 2 months after treatment. The repetition of 8 sessions of right IFG tDCS stimulation did not enhance the effects of mindfulness training on all these outcomes. Brown et $\mathrm{al}^{71}$ analyzed the EEG measures of 68 of the participants recruited in the first study. The visual cue presentation task measuring alcohol cue reactivity was administered during EEG sessions at baseline and post-treatment assessments. Follow-up EEG measures were also conducted 2 months after treatment. Conversely to the results obtained through the MBRP sessions, additional EEG measures showed no impact of tDCS either on the decrease of the late positive potential (ie, electrophysiological index of increased sensitivity towards alcohol-related cues) or on craving in response to alcohol-related images. Both studies showed a large sample size, obtained a good quality score (86\% and 93\%, respectively) and measured a large range of outcomes through behavioral and EEG measures. Table 2 summarizes the positive effects that tDCS had on the different outcomes measured in the aforementioned studies.

\section{Discussion}

The neurocognitive consequences of alcohol consumption are widespread in SAUD, ${ }^{3,4}$ and developing efficient rehabilitation programs to overcome these impairments is thus a priority. tDCS has emerged as a promising tool to address this issue. It has an established efficiency to improve cognitive abilities in other psychiatric populations, ${ }^{29}$ where it is commonly used as a complementary intervention tool with clear clinical guidelines. ${ }^{72}$ A growing interest has arisen toward its application in SAUD, but the number of studies exploring the beneficial effects of tDCS on SAUD symptoms is restricted, hampering to obtain clear conclusions regarding the conditions of its clinical implementation. Overall, the present review underlined the good methodological quality of most studies using tDCS in SAUD, thus ensuring the trustworthiness of their results. In this context, we highlighted the globally positive tDCS effects in this clinical population. Of note, these are in striking contradiction with studies among subclinical populations 


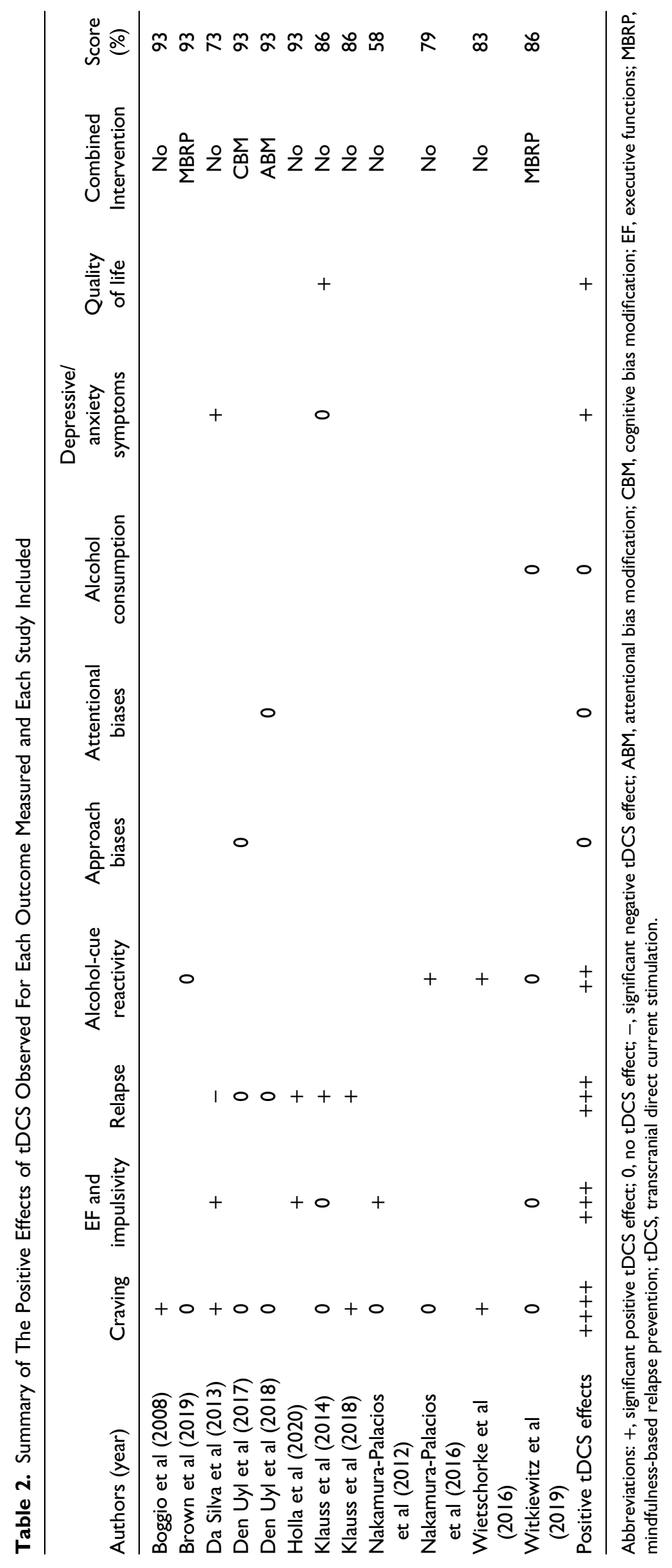


(eg, heavy or binge drinkers), coherently showing a lack of tDCS efficiency. ${ }^{73-75}$ tDCS thus does not constitute a reliable prevention or prophylactic tool, at least among adolescents and young adults presenting limited cognitive deficits and low motivation to change their drinking habits. Conversely, we suggest that it could constitute a useful adjunctive tool in the improvement of SAUD symptoms such as executive dysfunction or high level of subjective craving.

Indeed, results from studies using tDCS as a stand-alone treatment for SAUD showed positive effects and/or trends on alcohol-related symptoms, namely reduction of craving or relapse rate, executive functioning improvement, or enhancement of frontal activity. Nevertheless, we also highlighted inconsistencies across studies with high-methodological quality, starting with the fact that DLPFC stimulation reduced craving or improved frontal functions in some studies ${ }^{49,62,63}$ while it had no effect on others. ${ }^{57}$ In addition, according to the location of the anode over the left/right DLPFC, the relapse rate could be reduced ${ }^{57,62}$ or surprisingly increased. ${ }^{55}$ Studies using tDCS combined with other cognitive interventions, while all showing a strong methodology, did not observe any additive effect of tDCS on craving, executive functioning, cue reactivity, alcohol reduction or alcohol-related biases. ${ }^{66,67,70,71}$

As suggested earlier, ${ }^{38,76}$ these mixed results could be partly explained not only by sample heterogeneity (eg, SAUD duration and readiness to change) across studies, but also by the discrepancies in stimulation parameters. Besides the impact that electrode montages can have on the relapse rate when stimulating the DLPFC, electrodes size also matters, as larger cathodes (100 rather than $35 \mathrm{~cm}^{2}$ ) are associated with smaller effects on SAUD symptoms. ${ }^{66,67}$ Regarding the stimulation location, most studies have applied tDCS on the DLPFC, identifying this region as predominant in problematic alcohol use, or as being implicated in the underlying cognitive deficits. Beyond the influence of anode location, cathode placement, either on the contralateral DLPFC ${ }^{48,57,58,62,63,66,67}$ or on the contralateral shoulder $^{53,55}$ could also affect the outputs. The baseline impairment level has been overlooked by most studies, as 10 used a between-subject design rather than a within-subject design, while the latter allows to consider the individual baseline level and minimize the impact of interindividual differences. Therefore, inconsistencies across studies might be related to the variations across samples regarding preexisting cognitive deficits, as patients with limited or even no cognitive dysfunctions will obviously have low tDCS-related improvements. Finally, an offline task protocol has been preferred over an online protocol by most studies, while the latter would enhance performance outcomes through a synergistic effect. ${ }^{77}$ Indeed, a larger impact of tDCS is observed when the participant is involved, throughout the stimulation session, in a cognitive task. ${ }^{48}$ However, the 4 studies that attempted to combine tDCS with cognitive interventions in SAUD have used tasks involving the retraining of automatic processes (ie, attentional alcohol-related biases) ${ }^{66,67}$ or mindfulness. ${ }^{70,71}$ Although these interventions showed encouraging results at the behavioral level, ${ }^{78}$ these tasks, recruiting a low level of cognitive resources, often reach a ceiling effect which prevents tDCS stimulation from any further performance improvement, and do not involve the recruitment of frontal brain areas. It is therefore difficult to draw conclusions on the effectiveness of this combination based on the available results.

Besides stimulation parameters, the mixed results observed in SAUD could be related to inconsistencies regarding the measured outcomes. Indeed, studies assessed a large range of variables (eg, executive functioning, craving, cue reactivity, relapse, and alcohol consumption) and rarely evaluated the same outcomes. Even the evaluation of self-reported craving, the most frequently assessed variable, highly differed across studies regarding the questionnaires administered. Another issue related to craving assessment is its low level at baseline among patients with SAUD under detoxification, which could have induced a floor effect and hampered the observation of the tDCS effect on craving. The promising findings from Boggio et $\mathrm{al}^{49}$ showing a reduction of craving after a single tDCS session could be explained by the cue-provoked craving induced, resulting in higher baseline levels than in other studies. Finally, the available data regarding tDCS in SAUD might have been affected by a publication bias, leading us to overestimate the positive effects of tDCS.

Despite these limits, the current state of the art regarding tDCS in SAUD can lead to the following clinical recommendations. The most efficient way to improve the therapeutic settings in SAUD would be to combine cognitive remediation therapy and tDCS. Nevertheless, the combination of these interventions has to follow several principles (Figure 2):

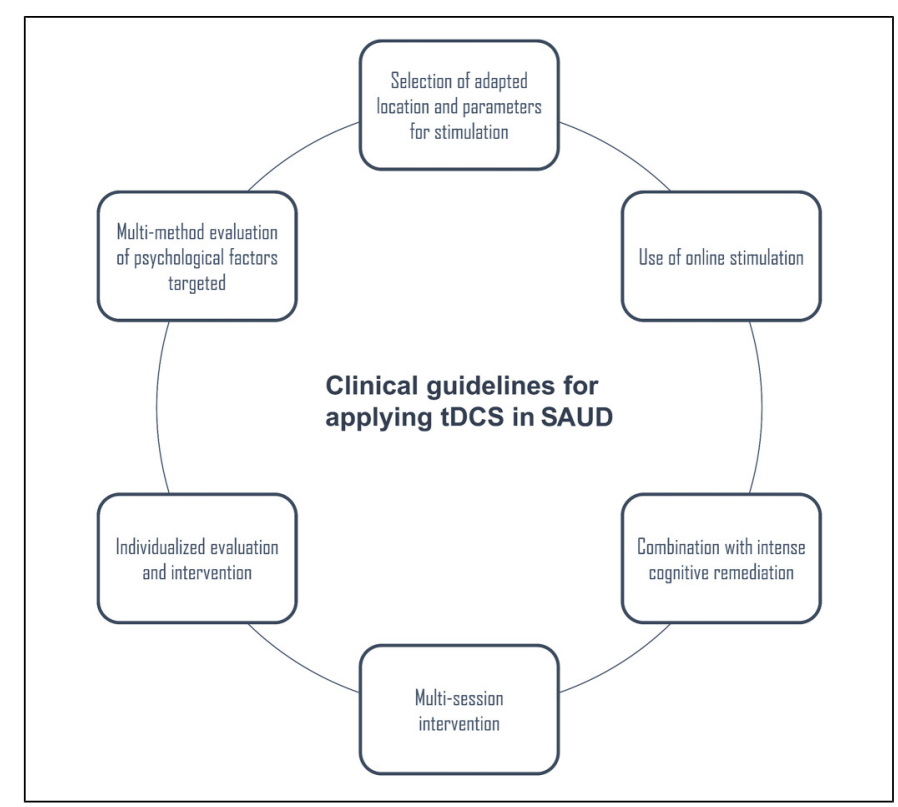

Figure 2. Proposal of evidence-based clinical guidelines for the application of tDCS in populations with SAUD.

Abbreviations: tDCS, transcranial direct current stimulation; SAUD, severe alcohol use disorder. 
1. Selecting adapted location and parameters for tDCS stimulation: the DLPFC is the most efficient area to be targeted by tDCS stimulation, as it can improve cognitive functioning and behavioral control, as well as reduce craving. Moreover, stimulation with high intensity (ie, $2 \mathrm{~mA}$ ) on a reduced surface should be preferred.

2. Using online stimulation: The effect of stimulation should be improved by privileging an online task protocol (ie, tDCS stimulation performs simultaneously with cognitive tasks), as it allows to benefit from synergistic effects. ${ }^{48,77}$

3. Combining tDCS with intense cognitive remediation: To reach higher efficiency, tDCS should be coupled with training tasks requiring high-cognitive activation and presenting parallel versions of the tasks. ${ }^{79,80}$ Indeed, previous studies combining tDCS with $\mathrm{CBM}^{67,68}$ often reached a ceiling effect due to low task difficulty and the absence of alternative versions. This could partly explain why the reduction of the bias was not enhanced by tDCS.

4. Proposing a multisession intervention: Multiple and repeated sessions of tDCS and cognitive training should be proposed to increase the behavioral and brain impact of tDCS, and to determine the long-term evolution of this impact on clinical variables.

5. Performing individualized evaluation/intervention: As tDCS can only be efficient among patients presenting a baseline deficit on the targeted processes, clinicians should systematically, before starting the therapeutic protocol, evaluate whether each patient actually presents a deficit in the cognitive functions targeted. The extent of cognitive deficits or craving indeed varies across patients. ${ }^{81}$ Moreover, the rehabilitation program should be individualized and personalized by offering patienttailored training. ${ }^{10}$ In the same manner, within-subject designs should be used to determine the beneficial effects of tDCS, in consideration of the high interindividual heterogeneity between patients at baseline; ${ }^{82}$

6. Capitalizing on a multimethod evaluation of the psychological factors targeted: To properly measure tDCS impact, the cognitive (eg, inhibition) or alcohol-related (eg, craving) variables targeted should be precisely determined beforehand and reliably measured before/after the intervention, ideally using a multimethod assessment (eg, combining self-reported measures with cue reactivity ones to evaluate craving).

\section{Conclusions}

The heterogeneous results highlighted by our review might partly be explained by sample characteristics (eg, low initial craving levels or low readiness to change), inconsistencies in stimulation parameters (eg, localization, density, tDCS design, and protocol), the variability of clinical outcome variables (eg, self-reported questionnaires of craving), and the nature of the cognitive processes targeted (eg, automatic processes such as cognitive biases). Despite these discrepancies, we consider that enough empirical evidence is available to apply tDCS in current clinical settings, providing the intervention relies on current knowledge by following the 6 above-mentioned principles. tDCS should also be combined with cognitive remediation and individualized evaluation/stimulation. However, further controlled clinical trials are required to support the efficiency of this procedure and to establish it as a widely applied clinical routine.

\section{Author contribution}

Conceptualization: VD and ZB; Methodology: PM and ZB; Selection procedure: PM and ZB; Methodological quality assessment: PM, ZB and VD; Writing - Original Draft Preparation: ZB; Writing - Review and Editing: VD and PM.

\section{Declaration of Conflicting Interests}

The authors declared no potential conflicts of interest with respect to the research, authorship and/or publication of this article.

\section{Funding}

The authors disclosed receipt of the following financial support for the research, authorship, and/or publication of this article: This work was supported by the Fonds De La Recherche Scientifique-FNRS.

\section{Supplemental Material}

Supplemental material for this article is available online.

\section{ORCID iDs}

Zoé Bollen (iD https://orcid.org/0000-0001-6137-0679

Pierre Maurage (iD https://orcid.org/0000-0003-0197-0810

\section{References}

1. Navarro HJ, Doran CM, Shakeshaft AP. Measuring costs of alcohol harm to others: a review of the literature. Drug Alcohol Depend. 2011;114(2-3):87-99. doi:10.1016/j.drugalcdep.2010. 11.009

2. Rehm J, Mathers C, Popova S, Thavorncharoensap M, Teerawattananon Y, Patra J. Global burden of disease and injury and economic cost attributable to alcohol use and alcoholuse disorders. Lancet. 2009;373(9682):2223-2233. doi:10.1016/ S0140-6736(09)60746-7

3. Stavro K, Pelletier J, Potvin S. Widespread and sustained cognitive deficits in alcoholism: a meta-analysis: alcoholism and cognition. Addict Biol. 2013;18(2):203-213. doi:10.1111/j.1369-1600. 2011.00418.x

4. Bühler M, Mann K. Alcohol and the human brain: a systematic review of different neuroimaging methods. Alcohol Clin Exp Res. 2011;35(10):1771-1793. doi:10.1111/j.1530-0277.2011. 01540.x

5. Nutt DJ, King LA, Phillips LD. Drug harms in the UK: a multicriteria decision analysis. Lancet. 2010;376(9752):1558-1565. doi:10.1016/S0140-6736(10)61462-6 
6. Rehm J, Shield KD, Gmel G, Rehm MX, Frick U. Modeling the impact of alcohol dependence on mortality burden and the effect of available treatment interventions in the European Union. Eur Neuropsychopharmacol. 2013;23(2):89-97. doi:10. 1016/j.euroneuro.2012.08.001

7. Kohn R, Saxena S, Levav I, Saraceno B. The treatment gap in mental health care. Bull World Health Organ. 2004;82(11): 858-866.

8. Maisto SA, Hallgren KA, Roos CR, Witkiewitz K. Course of remission from and relapse to heavy drinking following outpatient treatment of alcohol use disorder. Drug Alcohol Depend. 2018;187:319-326. doi:10.1016/j.drugalcdep.2018.03.011

9. Rochat L, Khazaal Y. Cognitive remediation therapy of working memory in addictive disorders: an individualized, tailored, and recovery-oriented approach. Expert Rev Neurother. 2019;19(4): 285-287. doi:10.1080/14737175.2019.1591950

10. Rolland $\mathrm{B}$, D'Hondt $\mathrm{F}$, Montègue $\mathrm{S}$, et al. A patient-tailored evidence-based approach for developing early neuropsychological training programs in addiction settings. Neuropsychol Rev. 2019;29(1):103-115. doi:10.1007/s11065-018-9395-3

11. Blume AW, Marlatt AG. The role of executive cognitive functions in changing substance use: what we know and what we need to know. Ann Behav Med. 2009;37(2):117-125. doi:10.1007/ s12160-009-9093-8

12. Czapla M, Simon JJ, Richter B, et al. The impact of cognitive impairment and impulsivity on relapse of alcohol-dependent patients: implications for psychotherapeutic treatment: cognitive deficits, relapse and alcohol. Addict Biol. 2016;21(4):873-884. doi:10.1111/adb.12229

13. Durazzo TC, Gazdzinski S, Yeh PH, Meyerhoff DJ. Combined neuroimaging, neurocognitive and psychiatric factors to predict alcohol consumption following treatment for alcohol dependence. Alcohol Alcohol. 2008;43(6):683-691. doi:10.1093/alcalc/agn078

14. Gil-Hernandez S, Garcia-Moreno LM. Executive performance and dysexecutive symptoms in binge drinking adolescents. Alcohol. 2016;51:79-87. doi:10.1016/j.alcohol.2016.01.003

15. Le Berre AP, Fama R, Sullivan EV. Executive functions, memory, and social cognitive deficits and recovery in chronic alcoholism: a critical review to inform future research. Alcohol Clin Exp Res. 2017;41(8):1432-1443. doi:10.1111/acer.13431

16. Rubio G, Jiménez M, Rodríguez-Jiménez R, et al. The role of behavioral impulsivity in the development of alcohol dependence: a 4-year follow-up study. Alcohol Clin Exp Res. 2008;32(9): 1681-1687. doi:10.1111/j.1530-0277.2008.00746.x

17. Salas-Gomez D, Fernandez-Gorgojo M, Pozueta A, et al. Binge drinking in young university students is associated with alterations in executive functions related to their starting age. PLoS One. 2016;11(11):e0166834. doi:10.1371/journal.pone.0166834

18. Domínguez-Salas S, Díaz-Batanero C, Lozano-Rojas OM, Verdejo-García A. Impact of general cognition and executive function deficits on addiction treatment outcomes: systematic review and discussion of neurocognitive pathways. Neurosci Biobehav Rev. 2016;71:772-801. doi:10.1016/j.neubiorev.2016. 09.030

19. Keshavan MS, Vinogradov S, Rumsey J, Sherrill J, Wagner A. Cognitive training in mental disorders: update and future directions. Am J Psychiatry. 2014;171(5):510-522. doi:10.1176/appi. ajp.2013.13081075

20. Rupp CI, Kemmler G, Kurz M, Hinterhuber H, Fleischhacker WW. Cognitive remediation therapy during treatment for alcohol dependence. J Stud Alcohol Drugs. 2012;73(4):625-634. doi:10.15288/jsad.2012.73.625

21. Bari A, DiCesare J, Babayan D, Runcie M, Sparks H, Wilson B. Neuromodulation for substance addiction in human subjects: a review. Neurosci Biobehav Rev. 2018;95:33-43. doi:10.1016/j. neubiorev.2018.09.013

22. Brunoni AR, Boggio PS, Ferrucci R, Priori A, Fregni F. Transcranial direct current stimulation: challenges, opportunities, and impact on psychiatry and neurorehabilitation. Front Psychiatry. 2013;4:19. doi:10.3389/fpsyt.2013.00019

23. Nitsche MA, Cohen LG, Wassermann EM, et al. Transcranial direct current stimulation: state of the art 2008. Brain Stimul. 2008;1(3):206-223. doi:10.1016/j.brs.2008.06.004

24. Nitsche MA, Paulus W. Excitability changes induced in the human motor cortex by weak transcranial direct current stimulation. J Physiol. 2000;527(3):633-639. doi:10.1111/j.1469-7793. 2000.t01-1-00633.x

25. Nitsche MA, Paulus W. Sustained excitability elevations induced by transcranial DC motor cortex stimulation in humans. Neurology. 2001;57(10):1899-1901. doi:10.1212/WNL.57.10. 1899

26. Nitsche MA, Fricke K, Henschke U, et al. Pharmacological modulation of cortical excitability shifts induced by transcranial direct current stimulation in humans. J Physiol. 2003;553(1): 293-301. doi:10.1113/jphysiol.2003.049916

27. Jacobson L, Koslowsky M, Lavidor M. tDCS polarity effects in motor and cognitive domains: a meta-analytical review. Exp Brain Res. 2012;216(1):1-10. doi:10.1007/s00221-011-2891-9

28. Thibaut A, Chatelle C, Gosseries O, Laureys S, Bruno MA. La stimulation transcrânienne à courant continu: un nouvel outil de neurostimulation. Rev Neurol. 2013;169(2):108-120. doi:10. 1016/j.neurol.2012.05.008

29. Kuo MF, Paulus W, Nitsche MA. Therapeutic effects of noninvasive brain stimulation with direct currents (tDCS) in neuropsychiatric diseases. Neuroimage. 2014;85:948-960. doi:10. 1016/j.neuroimage.2013.05.117

30. Boggio PS, Rigonatti SP, Ribeiro RB, et al. A randomized, double-blind clinical trial on the efficacy of cortical direct current stimulation for the treatment of major depression. Int J Neuropsychopharmacol. 2008;11(2):249-254. doi:10.1017/ S1461145707007833

31. Fregni F, Boggio PS, Nitsche MA, Rigonatti SP, Pascual-Leone A. Cognitive effects of repeated sessions of transcranial direct current stimulation in patients with depression. Depress Anxiety. 2006;23(8):482-484. doi:10.1002/da.20201

32. Wolkenstein L, Plewnia C. Amelioration of cognitive control in depression by transcranial direct current stimulation. Biol Psychiatry. 2013;73(7):646-651. doi:10.1016/j.biopsych.2012. 10.010

33. Hoy KE, Arnold SL, Emonson MRL, Daskalakis ZJ, Fitzgerald PB. An investigation into the effects of tDCS dose on cognitive performance over time in patients with schizophrenia. Schizophr Res. 2014;155(1-3):96-100. doi:10.1016/j.schres.2014.03.006

34. Knechtel L, Thienel R, Cooper G, Case V, Schall U. Transcranial direct current stimulation of prefrontal cortex: an auditory event-related potential study in schizophrenia. Neurol Psychiatry Brain Res. 2014;20(4):102-106. doi:10.1016/j.npbr. 2014.10.002

35. Elsner B, Kugler J, Pohl M, Mehrholz J. Transcranial direct current stimulation (tDCS) for idiopathic Parkinson's disease. 
Cochrane Database Syst Rev. 2016;7(7):CD010916. doi:10.1002/ 14651858.CD010916.pub2

36. Fregni F, Boggio PS, Bermpohl F, et al. Immediate placebo effect in parkinson's disease - is the subjective relief accompanied by objective improvement? Eur Neurol. 2006;56(4): 222-229. doi:10.1159/000096490

37. Coles AS, Kozak K, George TP. A review of brain stimulation methods to treat substance use disorders: Brain Stimulation to Treat SUDs. Am J Addict. 2018;27(2):71-91. doi:10.1111/ajad. 12674

38. Lupi M, Martinotti G, Santacroce R, et al. Transcranial direct current stimulation in substance use disorders: a systematic review of scientific literature. $J$ ECT. 2017;33(3):203-209. doi:10.1097/YCT.0000000000000401

39. Azevedo CA, Mammis A. Neuromodulation therapies for alcohol addiction: a literature review: neuromodulation therapies for alcohol addiction. Neuromodulation. 2018;21(2):144-148. doi:10.1111/ner.12548

40. Naish KR, Vedelago L, MacKillop J, Amlung M. Effects of neuromodulation on cognitive performance in individuals exhibiting addictive behaviors: a systematic review. Drug Alcohol Depend. 2018;192:338-351. doi:10.1016/j.drugalcdep.2018.08.018

41. Salib AM, Ho A, Sussman E, Pendharkar A, Halpern C. Neuromodulatory treatments for alcohol use disorder: a review. Brain Sci. 2018;8(6):95. doi:10.3390/brainsci8060095

42. Lapenta OM, Marques LM, Rego GG, Comfort WE, Boggio PS. TDCS In addiction and impulse control disorders. $J$ ECT. 2018;34(3):182-192. doi:10.1097/YCT.0000000000000541

43. Mostafavi SA, Khaleghi A, Mohammadi MR. Noninvasive brain stimulation in alcohol craving: a systematic review and meta-analysis. Prog Neuro-psychopharmacol Biol Psychiatry. 2020;101:109938. doi:10.1016/j.pnpbp.2020.109938

44. Philip NS, Sorensen DO, McCalley DM, Hanlon CA. Non-invasive brain stimulation for alcohol use disorders: state of the art and future directions. Neurotherapeutics. 2020;17(1):116-126. doi:10.1007/ s13311-019-00780-x

45. National Heart, Lung, Blood Institute. Quality Assessment Tool for Controlled Intervention Studies. Available at National Heart, Lung, and Blood Institute; 2014.

46. Moher D, Liberati A, Tetzlaff J, Altman DG, The PRISMA Group. Preferred reporting items for systematic reviews and meta-analyses: the PRISMA statement. PLoS Med. 2009; 6(7):e1000097. doi:10. 1371/journal.pmed.1000097

47. Black MH, Chen NTM, Iyer KK, et al. Mechanisms of facial emotion recognition in autism spectrum disorders: insights from eye tracking and electroencephalography. Neurosci Biobehav Rev. 2017;80:488-515. doi:10.1016/j.neubiorev.2017.06.016

48. Elmasry J, Loo C, Martin D. A systematic review of transcranial electrical stimulation combined with cognitive training. Restor Neurol Neurosci. 2015;33(3):263-278. doi:10.3233/RNN-140473

49. Boggio PS, Sultani N, Fecteau S, et al. Prefrontal cortex modulation using transcranial DC stimulation reduces alcohol craving: a double-blind, sham-controlled study. Drug Alcohol Depend. 2008;92(1-3):55-60. doi:10.1016/j.drugalcdep.2007.06.011

50. Drummond DC, Phillips TS. Alcohol urges in alcohol-dependent drinkers: further validation of the alcohol urge questionnaire in an untreated community clinical population. Addiction. 2002;97(11):1465-1472. doi:10.1046/j.1360-0443.2002.00252.x

51. George MS, Anton RF, Bloomer C, et al. Activation of prefrontal cortex and anterior thalamus in alcoholic subjects on exposure to alcohol-specific cues. Arch Gen Psychiatry. 2001;58(4):345-352. doi:10.1001/archpsyc.58.4.345

52. Olbrich HM, Valerius G, Paris C, Hagenbuch F, Ebert D, Juengling FD. Brain activation during craving for alcohol measured by positron emission tomography. Aust $N$ Z J Psychiatry. 2006;40(2):171-178. doi:10.1080/j.1440-1614.2006.01765.x

53. Nakamura-Palacios EM, de Almeida Benevides MC, da Penha Zago-Gomes M, et al. Auditory event-related potentials (P3) and cognitive changes induced by frontal direct current stimulation in alcoholics according to Lesch alcoholism typology. Int J Neuropsychopharmacol. 2012;15(05):601-616. doi:10.1017/ S1461145711001040

54. Dubois B, Slachevsky A, Litvan I, Pillon B. The FAB: a frontal assessment battery at bedside. Neurology. 2000;55(11): 1621-1626. doi:10.1212/WNL.55.11.1621

55. da Silva MC, Conti CL, Klauss J, et al. Behavioral effects of transcranial direct current stimulation (tDCS) induced dorsolateral prefrontal cortex plasticity in alcohol dependence. J Physiol. 2013;107(6):493-502. doi:10.1016/j.jphysparis.2013.07.003

56. Tiffany ST, Wray JM. The clinical significance of drug craving: Tiffany \& Wray. Ann NY Acad Sci. 2012;1248(1):1-17. doi:10. 1111/j.1749-6632.2011.06298.x

57. Klauss J, Penido Pinheiro LC, Silva Merlo BL, et al. A randomized controlled trial of targeted prefrontal cortex modulation with tDCS in patients with alcohol dependence. Int J Neuropsychopharmacol. 2014;17(11):1793-1803. doi:10.1017/S1461145714000984

58. Nakamura-Palacios EM, Lopes IBC, Souza RA, et al. Ventral medial prefrontal cortex (vmPFC) as a target of the dorsolateral prefrontal modulation by transcranial direct current stimulation (tDCS) in drug addiction. J Neural Transm. 2016;123(10): 1179-1194. doi:10.1007/s00702-016-1559-9

59. Bzdok D, Langner R, Schilbach L, et al. Segregation of the human medial prefrontal cortex in social cognition. Front Hum Neurosci. 2013;7:232. doi:10.3389/fnhum.2013.00232

60. Seo D, Lacadie CM, Tuit K, Hong KI, Constable RT, Sinha R. Disrupted ventromedial prefrontal function, alcohol craving, and subsequent relapse risk. JAMA Psychiatry. 2013;70(7):727-739. doi:10.1001/jamapsychiatry.2013.762

61. Verdejo-García A, Bechara A. A somatic marker theory of addiction. Neuropharmacology. 2009;56:48-62. doi:10.1016/ j.neuropharm.2008.07.035

62. Klauss J, Anders QS, Felippe LV, Nitsche MA, Nakamura-Palacios EM. Multiple sessions of transcranial direct current stimulation (tdcs) reduced craving and relapses for alcohol use: a randomized placebo-controlled trial in alcohol use disorder. Front Pharmacol. 2018;9:716. doi:10.3389/fphar.2018.00716

63. Wietschorke K, Lippold J, Jacob C, Polak T, Herrmann MJ. Transcranial direct current stimulation of the prefrontal cortex reduces cue-reactivity in alcohol-dependent patients. $J$ Neural Transm. 2016;123(10):1173-1178. doi:10.1007/s00702-016-1541-6

64. Vrana SR, Lang PJ. Fear imagery and the startle-probe reflex. $J$ Abnorm Psychol. 1990;99(2):189-197. doi:10.1037/0021-843X. 99.2.189

65. Holla B, Biswal J, Ramesh V, et al. Effect of prefrontal tDCS on resting brain fMRI graph measures in alcohol Use disorders: a randomized, double-blind, sham-controlled study. Prog Neuropsychopharmacol Biol Psychiatry. 2020; 102:109950. doi:10.1016/j.pnpbp.2020.109950

66. den Uyl TE, Gladwin TE, Rinck M, Lindenmeyer J, Wiers RW. A clinical trial with combined transcranial direct current stimulation 
and alcohol approach bias retraining: clinical trial tDCS and CBM. Addict Biol. 2017;22(6):1632-1640. doi:10.1111/adb. 12463

67. den Uyl TE, Gladwin TE, Lindenmeyer J, Wiers RW. A clinical trial with combined transcranial direct current stimulation and attentional bias modification in alcohol-dependent patients. Alcohol Clin Exp Res. 2018;42(10):1961-1969. doi:10.1111/acer.13841

68. Eberl C, Wiers RW, Pawelczack S, Rinck M, Becker ES, Lindenmeyer J. Approach bias modification in alcohol dependence: do clinical effects replicate and for whom does it work best? Dev Cogn Neurosci. 2013;4:38-51. doi:10.1016/j.den.2012.11.002

69. Wiers RW, Eberl C, Rinck M, Becker ES, Lindenmeyer J. Retraining automatic action tendencies changes alcoholic patients' approach bias for alcohol and improves treatment outcome. Psychol Sci. 2011;22(4):490-497. doi:10.1177/0956797611400615

70. Witkiewitz K, Stein ER, Votaw VR, et al. Mindfulness-based relapse prevention and transcranial direct current stimulation to reduce heavy drinking: a double-blind sham-controlled randomized trial. Alcohol Clin Exp Res. 2019;43(6):1296-1307. doi:10. 1111/acer.14053

71. Brown DR, Jackson TCJ, Claus ED, et al. Decreases in the late positive potential to alcohol images among alcohol treatment seekers following mindfulness-based relapse prevention. Alcohol Alcohol. 2020;55(1):78-85. doi:10.1093/alcalc/agz096

72. Palm U, Hasan A, Strube W, Padberg F. tDCS for the treatment of depression: a comprehensive review. Eur Arch Psychiatry Clin Neurosci. 2016;266(8):681-694. doi:10.1007/s00406-016-0674-9

73. Claus ED, Klimaj SD, Chavez R, Martinez AD, Clark VP. A randomized trial of combined tDCS over right inferior frontal cortex and cognitive bias modification: null effects on drinking and alcohol approach bias. Alcohol Clin Exp Res. 2019;43(7): 1591-1599. doi:10.1111/acer.14111

74. den Uyl TE, Gladwin TE, Wiers RW. Transcranial direct current stimulation, implicit alcohol associations and craving. Biol Psychol. 2015;105:37-42. doi:10.1016/j.biopsycho.2014.12.004
75. den Uyl TE, Gladwin TE, Wiers RW. Electrophysiological and behavioral effects of combined transcranial direct current stimulation and alcohol approach bias retraining in hazardous drinkers. Alcohol Clin Exp Res. 2016;40(10):2124-2133. doi:10.1111/ acer. 13171

76. Hone-Blanchet A, Ciraulo DA, Pascual-Leone A, Fecteau S. Noninvasive brain stimulation to suppress craving in substance use disorders: review of human evidence and methodological considerations for future work. Neurosci Biobehav Rev. 2015;59:184-200. doi:10.1016/j.neubiorev.2015.10.001

77. Dedoncker J, Brunoni AR, Baeken C, Vanderhasselt MA. A systematic review and meta-analysis of the effects of transcranial direct current stimulation (tDCS) over the dorsolateral prefrontal cortex in healthy and neuropsychiatric samples: influence of stimulation parameters. Brain Stimul. 2016;9(4): 501-517. doi:10.1016/j.brs.2016.04.006

78. Manning V, Staiger PK, Hall K, et al. Cognitive bias modification training during inpatient alcohol detoxification reduces early relapse: a randomized controlled trial. Alcohol Clin Exp Res. 2016;40(9):2011-2019. doi:10.1111/acer.13163

79. Pope PA, Miall RC. Task-specific facilitation of cognition by cathodal transcranial direct current stimulation of the cerebellum. Brain Stimul. 2012;5(2):84-94. doi:10.1016/j.brs.2012.03.006

80. Popescu T, Krause B, Terhune DB, et al. Transcranial random noise stimulation mitigates increased difficulty in an arithmetic learning task. Neuropsychologia. 2016;81:255-264. doi:10.1016/ j.neuropsychologia.2015.12.028

81. Alarcon R, Nalpas B, Pelletier S, Perney P. MoCA as a screening tool of neuropsychological deficits in alcohol-dependent patients. Alcohol Clin Exp Res. 2015;39(6):1042-1048. doi:10.1111/acer. 12734

82. Luigjes J, Segrave R, de Joode N, Figee M, Denys D. Efficacy of invasive and non-invasive brain modulation interventions for addiction. Neuropsychol Rev. 2018;29(1):116-138. doi:10.1007/ s11065-018-9393-5 